\title{
A POPULATION CYTOGENETIC STUDY OF A COMMON FRAGILE SITE, fra(3)(p14), IN A HEALTHY POPULATION
}

\author{
Motoi Murata, ${ }^{1}$ Mikako Otsuka, ${ }^{1}$ Yukiko Hayakawa, ${ }^{1}$ \\ Ei-ichi TakaHASHI, ${ }^{2}$ Hideo TsuJI, ${ }^{2}$ and Tada-aki HoR ${ }^{2}$ \\ ${ }^{1}$ Division of Epidemiology, Chiba Cancer Center Research Institute, \\ Chiba 280, Japan \\ ${ }^{2}$ Division of Genetics, National Institute of Radiological Sciences, \\ Chiba 260, Japan
}

\begin{abstract}
Summary A population survey of a common folate-sensitive fragile site, fra(3)(p14), was carried out on PHA-stimulated peripheral lymphocytes of 1,078 healthy subjects. $\operatorname{Fra}(3)(\mathrm{p} 14)$ was expressed more frequently in the younger than in the older, and in males than in females. It also showed some seasonal variation. The age difference of the expression frequency was also observed by aphidicolin treatment. A positive correlation of the expression frequency was found, though not so strongly, between both culture conditions of folate deprivation and aphidicolin treatment, These findings suggest that the inter-individual variation in the expressivity of common fragile sites is not only ascribable to chance, but to some physiological conditions of blood donors such as response rate of lymphocyte to PHA stimulation and blood concentration of folic acid.
\end{abstract}

Key Words fra(3)(p14), folate deficiency, aphidicolin, cell cycle, age

\section{INTRODUCTION}

Many common fragile sites have been identified on human chromosomes (Hecht, 1988). They are expressed in cultured lymphocytes under certain culture conditions, such as folate deprivation and aphidicolin (APC) treatment (Sutherland and Hecht, 1985). They are defined as the common because we can find no special class of a population whose lymphocyte chromosomes could express a specific common fragile site, either wholly or not at any rate. However, as we proceed a fragile site test on a number of subjects, a wide inter-individual variation can be seen in their expressivity. Whether this variation is no more than a random fluctua- 
tion or might be due to some background biological mechanisms is the point of interest of the present study.

Regarding this question, Craig-Holmes et al. (1987) observed that expression of common fragile sites by APC treatment was incompatible among three consecutive samplings from the same three persons. Smeets et al. (1986) also demonstrated that the expression of fra(3)(p14) with the folate deficient condition was significantly disagreed between two consecutive samples in three of the six cases. They also clarified that its expression was randomly distributed between the two homologous of chromosome 3 . These findings strongly suggest that the observed variation is primarily owing to sampling differences. But, as yet, possible factors which may be relevant to the expression of common fragile sites have not been extensively studied. From this point of view, a large-scale survey study was conducted on the variable expression of the common fragile site in a healthy population. For this study, only fra(3)(pl4) was chosen because it not only is observable most frequently of all the common fragile sites but also can easily be identified without any chromosome banding.

\section{MATERIALS AND METHODS}

Study subjects. Present survey was first conducted on a population of 963 healthy blood donors ( 609 males and 354 females). Frequency of heritable fragile sites in this population was previously reported (Takahashi et al., 1988). Their ages were distributed from high teens to fifties, with a mean of 31 years. The fragile site examination was performed from January, 1985 to September, 1987, by using only folate deficient culture. Later we considered it necessary to succeed this survey, including the test of common fragile site induced by APC treatment. From November, 1989 to August, 1990, 115 healthy blood donors (54 males and 61 females) were newly added to the survey and the culture conditions of both folate deprivation and APC treatment were used. For the latter group, the cell cycle test of cultured lymphocytes was also conducted. Furthermore, a part of these newly tested donors (12 males and 20 females), plasma concentration of folic acid was examined by requesting a center of laboratory tests (Special Reference Laboratory, Tokyo).

Thus, a total of 1,078 donors (663 males and 415 females) were enrolled for the examination of fra( 3 (p14) in folate deficiency and 108 (49 males and 59 females) in APC treatment.

Examination of fragile site expression. For examining the expression of fragile site, a $72 \mathrm{hr}$ whole blood culture was conducted as follows. In each culture, 0.5 $\mathrm{ml}$ of heparinized whole blood sample was added in $4.5 \mathrm{ml}$ of culture medium and incubated in a $\mathrm{CO}_{2}(5 \%)$ incubator at $37^{\circ} \mathrm{C}$. The culture condition for folate deprivation was set up by using folic acid- and thymidine-free Ham's F10 medium (M-F10, Gibco) containing 5\% fetal calf serum (FCS, Difco) and $2 \%$ phytohemag- 
glutinin (PHA, Wellcome). According to this assay system, folic acid deprivation is rather incomplete, the final concentration being around $0.8 \mathrm{ng} / \mathrm{ml}$ which is about one-fifth of that in blood serum. APC induced expression was examined by culturing lymphocytes in PRMI 1640 medium (Nissui) containing $20 \%$ FCS and $2 \%$ PHA, and $0.1 \mu \mathrm{M}$ aphidicolin (Wako) was added for the last $24 \mathrm{hr}$ (Glover et al., 1984). To examine the background level of expression, the culture without APC was made. After $72 \mathrm{hr}$, chromosome preparations were made by a standard method following colchicine treatment $(2 \mathrm{hr})$, hypotonic treatment $(0.075 \mathrm{M} \mathrm{KCl})$, ethanol-acetic acid (3:1) fixation and air-drying. Fifty metaphase plates were scored for each culture condition on each subject and expression of fra(3)(p14) was counted. Expression frequency was given by its number in 50 cells.

Examination of cell cycle progression. To test whether the degree of cell proliferation of cultured lymphocytes is related to varying expressions of the common fragile site, progression of cell cycles was examined (Morimoto et al., 1983). For this, peripheral blood samples from 96 donors were cultured with bromodeoxyuridine (BrdU, $7 \mu \mathrm{g} / \mathrm{ml}$, Sigma) for $72 \mathrm{hr}$. Chromosomes labeled with BrdU were stained with $5 \mu \mathrm{g} / \mathrm{ml}$ Hoechst 33258 in phosphate-buffer ( $\mathrm{pH} \mathrm{6.8)}$ ) for $30 \mathrm{~min}$, washed in the buffer, covered with a cover-slip and exposed to $360 \mathrm{~nm}$ light from fluorescence black light tubes for $15 \mathrm{~min}$ on a hot-plate at $60^{\circ} \mathrm{C}$. The slides were stained with $3 \%$ Giemsa for $3 \mathrm{~min}$. According to the pattern of differential staining of sister chromatids, metaphases were classified into M1, M2, and M3 + as having undergone one, two, and three or more cell cycles, respectively. For each subject, 200 metaphases were scored and the proportion of $\mathrm{M} 3+$ cells was calculated as an index of cell cycle progression.

For eight subjects, both the expression of fra(3)(p14) and the cell cycle progression were examined in different culture times, by harvesting cells successively at 48, 72, and $96 \mathrm{hr}$. Furthermore, only at $96 \mathrm{hr}$, a final concentration of folic acid in the culture medium was measured.

\section{RESULTS}

\section{Expression of fra(3) ( $p 14)$ by folate deficiency}

Frequency distribution of the number of fra(3)(p14) expressed by the folate deprivation is shown in Table 1 . The expression frequency was widely distributed from zero to 10 or more in 50 cells. About $30-40 \%$ of samples were of non-expressed. This must be due to the culture condition of incomplete folate deficiency. It appeared that the observed variation is primarily owing to the random fluctuation. However the distribution does not fit to the Poisson expectation $\left(\chi^{2}=91.7\right.$, d.f. $=6$, $\mathrm{p}<0.001$ ), but rather to a negative binomial distribution.

Males showed slightly higher expressivity, on the average, than females as obviously seen in Fig. 1. This sex difference was more remarkable in younger than in older age classes. Thus, the negative regression of the expression frequency 
M. MURATA et al.

Table 1. Frequency distribution of number of $\mathrm{fra}(3)(\mathrm{p} 14)$ in 50 metaphase cells cultured with folate deficient M-F10 medium.

\begin{tabular}{|c|c|c|c|}
\hline \multirow{2}{*}{$\begin{array}{l}\text { Number of } \\
\text { fra(3)(p14) }\end{array}$} & \multicolumn{3}{|c|}{ Number of cases } \\
\hline & Male & Female & Total \\
\hline 0 & 197 & 151 & 348 \\
\hline 1 & 153 & 94 & 247 \\
\hline 2 & 117 & 82 & 199 \\
\hline 3 & 62 & 41 & 103 \\
\hline 4 & 50 & 21 & 71 \\
\hline 5 & 33 & 12 & 45 \\
\hline 6 & 15 & 4 & 19 \\
\hline 7 & 13 & 4 & 17 \\
\hline 8 & 1 & 1 & 12 \\
\hline 9 & 6 & 4 & 10 \\
\hline $10+$ & 6 & 1 & 7 \\
\hline Total & 663 & 415 & 1,078 \\
\hline
\end{tabular}

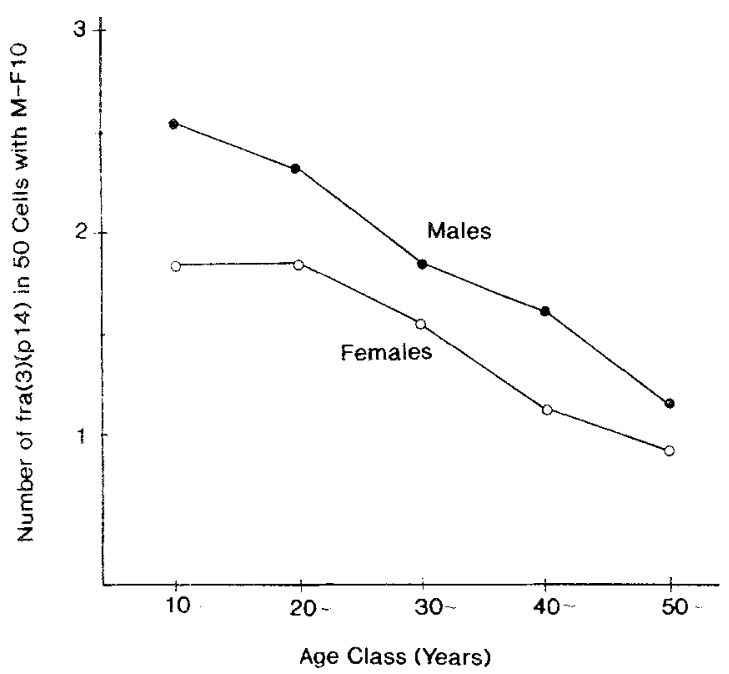

Fig. 1. Mean number of fra(3)(p14) in 50 cells with folate deficient culture in different sex- and age classes of a healthy population.

can be delineated against the donor's age in both sexes, but more steeply in males $(b=-0.038 \pm 0.008, p<0.001)$ than in females $(b=-0.026 \pm 0.007, p<0.001)$. Moreover, some sorts of seasonal variation were also observed in its expressivity, with the highest in November and the lowest in August (Fig. 2). These findings 


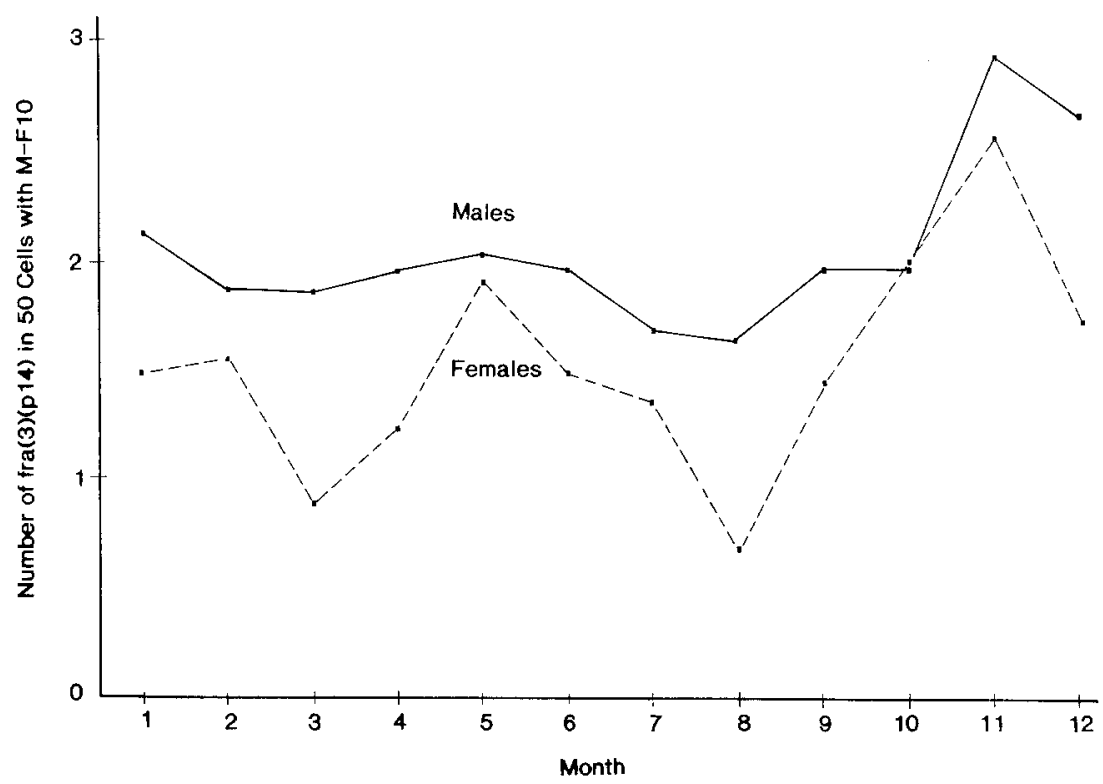

Fig. 2. Seasonal changes of the mean expression frequency of fra(3)(p14) in 50 cells with folate deficient culture of lymphocytes from healthy males (solid line) and females (dotted line).

apparently indicate that the expressivity with this culture condition is controlled at least partly by some physiological condition of donors.

Expression of fra (3) (p14) by $A P C$

The distribution of the expression frequency of fra(3)(p14) by APC is shown for both sexes in Table 2. Mean expressivity in 50 cells was $9.2 \pm 0.7$ in males and $10.1 \pm 0.7$ in females with no significant difference between the sexes. On the other hand, negative regression of the expressivity onto the age of the subjects was seen $(b=-0.10 \pm 0.04, p<0.01)$ (Fig. 3), similar to the result obtained in the folate deficient culture (Fig. 1).

Correlation between the expression frequencies with the M-F10 culture and with the APC treatment was significant $(r=0.23$, d.f. $=105, p<0.05$ ) (Fig. 4). However, the correlation becomes non-significant after adjusting for the donor's age (partial correlation coefficient $=0.18$, d.f. $=104,0.05<\mathrm{p}<0.10$ ), indicating that certain age-related factors may be commonly involved in expressions of fra(3) (p14) with both culture conditions.

\section{Cell cycle progression in lymphocyte culture}

Inter-individual variation in the cell proliferating rate of lymphocytes cultured in the normal medium is presented in Fig. 5 with the average proportion of M3+ cells and a confidence interval of one standard error in different age classes. As 
Table 2. Frequency distribution of number of fra(3)(p14) in 50 metaphase cells cultured with aphidicolin treatment.

\begin{tabular}{cccc}
\hline $\begin{array}{c}\text { Number of } \\
\text { fra }(3)(\mathrm{p} 14)\end{array}$ & \multicolumn{3}{c}{ Number of cases } \\
\cline { 2 - 4 } & Male & Female & Total \\
\hline $1-2$ & 2 & 2 & 4 \\
$3-4$ & 10 & 3 & 13 \\
$5-6$ & 7 & 6 & 13 \\
$7-8$ & 2 & 13 & 15 \\
$9-10$ & 8 & 9 & 17 \\
$11-12$ & 9 & 13 & 22 \\
$13-14$ & 4 & 6 & 10 \\
$15-16$ & 2 & 2 & 4 \\
$17-18$ & 3 & 3 & 6 \\
$19+$ & 2 & 2 & 4 \\
\hline Total & 49 & 59 & 108 \\
\hline
\end{tabular}

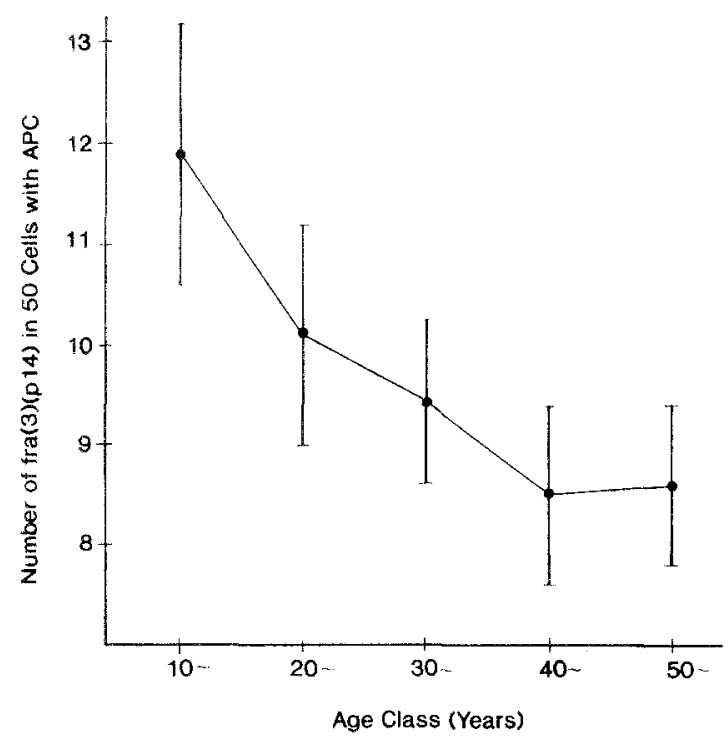

Fig. 3. Mean number of fra(3)(p14) in 50 cells with aphidicolin treatment in different age classes for males and females combined, with a confidence interval of one standard error.

a whole, the proportion varied very widely, ranging from 30 to $85 \%$. Even though a certain kind of non-linear regression against the donor's age is seen with a peak in twenties in both sexes, it was not statistically significant. Moreover, no correla- 


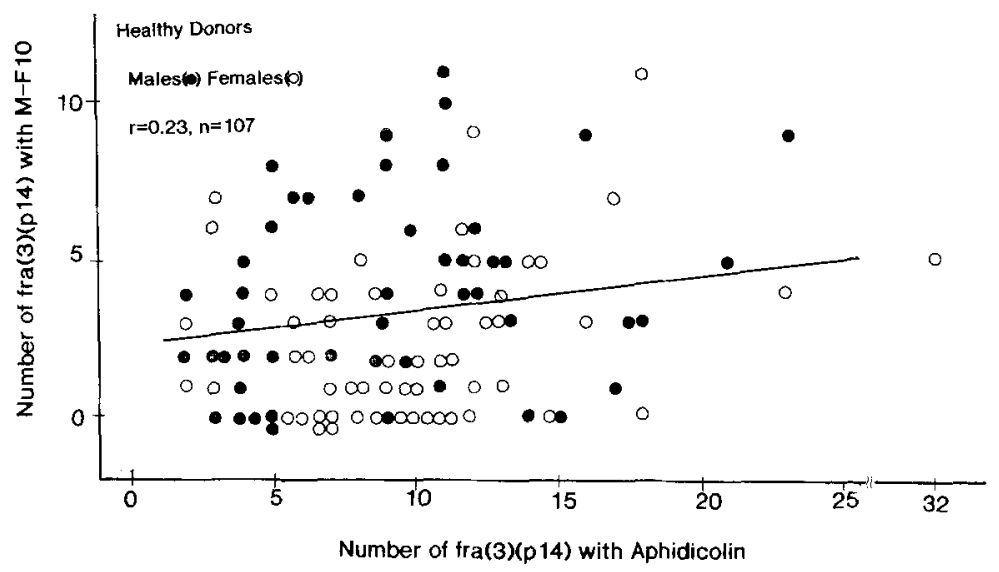

Fig. 4. Correlation between the expression frequencies of fra(3)(p14) with the folate deficient and the aphidicolin treated cultures, with a linear regression line.

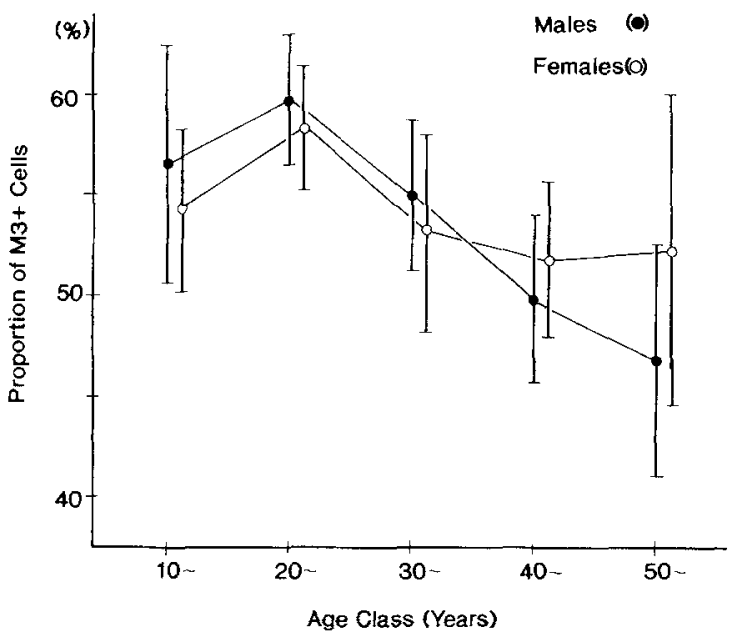

Fig. 5. Mean proportion of M3 + cells at $72 \mathrm{hr}$ of lymphocyte culture with RPMI 1640 medium in different sex- and age classes, with a confidence interval of one standard error.

tion was found between this trait and expression frequency of fra(3)(p14) in M-F10 culture (Fig. 6).

The expression frequency of fra(3)(p14) by folate deprivation as well as the progression of cell cycles is shown as a changing pattern in the consecutive days of lymphocyte culture (48, 72, and $96 \mathrm{hr}$ ) for each of 8 healthy donors (Fig. 7). It appears, on the average, that the expression of fra(3)(p14) should be enhanced in later stages of culture, indicating a positive effect of the cell cycle progression 


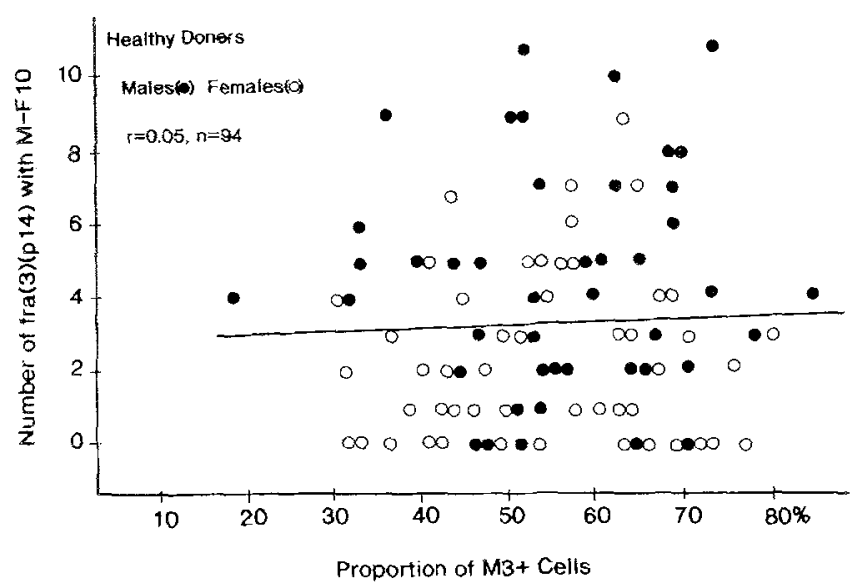

Fig. 6. Correlation between the expression frequency of fra(3)(p14) with folate deficient culture and the proportion of $\mathrm{M} 3+$ cells in cultures with normal medium, with a linear regression line.

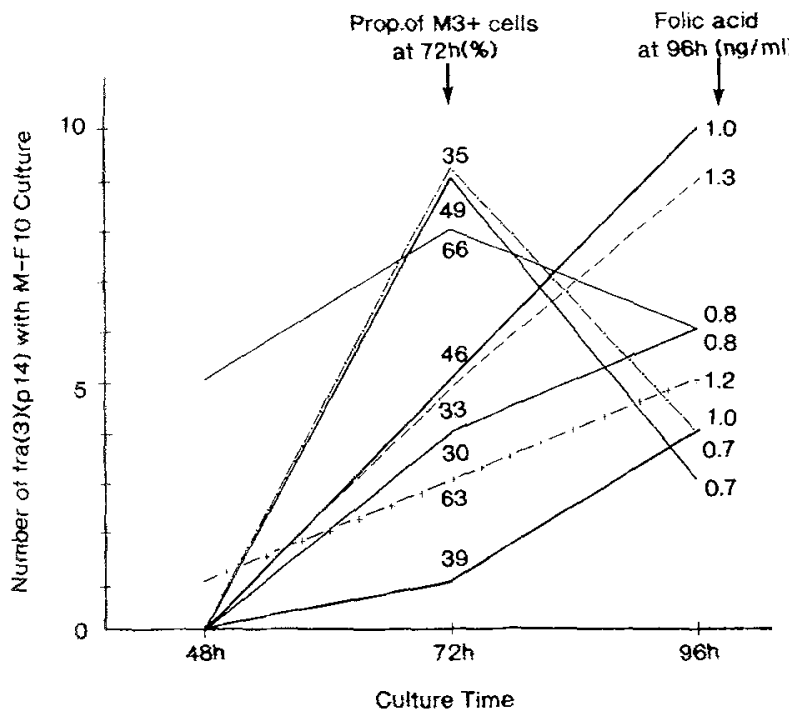

Fig. 7. Changing patterns of expressivity of fra(3)(p14) at different culture times for 8 sample subjects. Values shown at $72 \mathrm{hr}$ are the proportion of $\mathrm{M} 3+$ cells at this time of lymphocyte culture with RPMI 1640 medium. Values shown at $96 \mathrm{hr}$ indicate the final concentration of folic acid in the culture with M-F10 medium.

on the expressivity. This is contradictory to the above mentioned non-correlation between both traits (Fig. 6). In some cases, however, the expressivity was rather reduced in the 96 than in the $72 \mathrm{hr}$ culture. In these cases, the expression seems to be maximized in some intermediate culture times, implicating that there should 


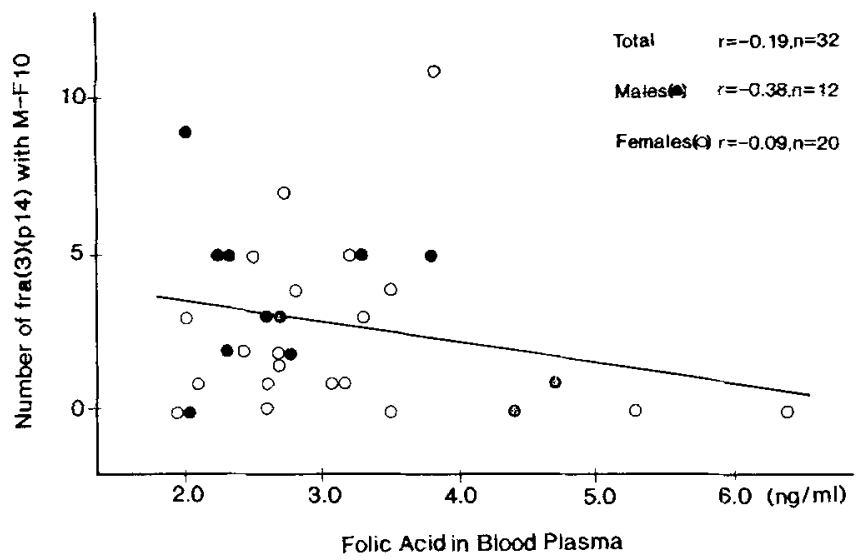

Fig. 8. Correlation between the expressivity of fra(3)(p14) in folate deficient culture and the plasma concentration of folate, with a linear regression line.

be a certain fraction of cells committed to express the common fragile site in peripheral lymphocytes. Those cases with a higher proportion of $\mathrm{M} 3+$ cells at 72 $\mathrm{hr}$ tended to express the fragile site earlier than those with lower one.

Measurements of the final concentration of folic acid in M-F10 at $96 \mathrm{hr}$ of culture varied from 0.7 to $1.3 \mathrm{ng} / \mathrm{ml}$ among these donors (Fig. 7). However this trait is not at all correlated with the expression of fra(3)(p14) either at $72 \mathrm{hr}$ or at $96 \mathrm{hr}$. Thus it is predicted that the expression of fragile site is affected with multiple factors, including both cell proliferation rate and folate concentration.

\section{Blood concentration of folic acid}

Measurements of folic acid in blood plasma of healthy donors were distributed from 2 to $6.4 \mathrm{ng} / \mathrm{ml}$, with means of $2.0 \pm 0.3$ in 12 males and $3.1 \pm 0.4$ in 20 females. There was no significant difference between the sexes. Correlation between this trait and the expressivity of fra(3)(p14) in M-F10 culture was shown in Fig. 8. Although there appears slightly a negative correlation between them, it is not significant.

\section{DISCUSSION}

With respect to the expressivity of common fragile sites, a few studies have been so far reported on a survey of a healthy human population (Smeets et al., 1986; Kähkönen, 1988). The present study was performed with a much larger number of subjects than them. It could only be proceeded by having employed an assay system which simply scores fra(3)(p14), neglecting all the other sites. We have chosen this site because it is the most frequently observed common fragile site on human chromosomes by any kinds of inducing culture condition. It is presently 
not conclusive whether any common fragile sites are expressed comparably with each other. Previous studies (Craig-Holmes et al., 1987; Rao et al., 1988a, b; Fuster et al., 1989) sometimes exhibited individuals whose lymphocytes express other common fragile sites more frequently than fra(3)(p14). Thus for the moment the findings obtained presently should be confined to be true for fra(3)(p14).

Results of the present study revealed that there should be a sizable amount of inter-individual variation in the expressivity of fra(3)(p14) in both the folate deficient and the APC treated cultures. Poisson-like distribution observed in the folate deficient culture was very similar to those reported by other authors (Smeets et al., 1986; Kähkönen, 1988), though the mean expressivity was about one-half of them, probably because of different kinds of culture condition. However, the distribution was somehow biased from the Poisson distribution, as was also reported by Kähkönen (1988). It thus seems that the expressivity of a common fragile site must be influenced not only by chance but also by certain kinds of physiological conditions of blood donors. Since there is no evidence of bi- or trimodality in the frequency distribution of the expressivity, any simple mode of hereditary factors should not be assumed. Negative evidence on the hereditary factor has already been reported by other authors (Sugio and Kuroki, 1989).

Most interesting finding is the sex- and age differences of the expressivity, which were not so clearly seen in the result of Smeets et al. (1986). In the present result, the expressivity was higher in males than in females under the folate deficient culture condition, but not by APC treatment. It was also higher in younger than in older subjects under both culture conditions, though more remarkably in the folate deprivation. Any sexual difference in terms of an induction rate of morphological changes of somatic chromosomes has been very occasionally reported. Dewdney et al. (1986) reported, without any interpretation, that base-line frequency of sister chromatid exchanges was slightly but significantly larger in females than males. D'Souza et al. (1988) also demonstrated that the frequencies of both sister chromatid exchanges and chromosomal aberrations such as gaps and breaks were higher in females than in males and that the difference was quite temarkable at the ovulatory and estrogenic stages of the women's menstrual cycle. Although these are an opposite of the present result of the preponderance in males, they at least suggest that some sort of steroid hormone must be involved in the process of cell proliferation or more directly in DNA replication.

The results of the cell cycle test indicate, though not significantly in a statistical sense, that lymphocytes from younger donors are more actively respond to the PHA stimulation and proliferate more rapidly than older ones (Fig. 5). This age difference is quite agreeable to the result of Tice et al. (1979), who studied cell kinetics of the cultured lymphocytes from four young and four old men and found the proliferation rate of lymphocytes from older men to be significantly worse than that from younger ones. Reduced response of lymphocytes from elderly subjects to certain mitogen stimulation has also been confirmed by several other authors 
(Murasko et al., 1987).

However, as opposed to our expectation, correlation of the fra(3)(p14) expressivity with the proportion of M3 + cells was not so straightforward (Fig. 6). It should be pointed out that the cell proliferation rates observed under the culture condition of normal medium may not be strictly comparable to the situation occurring in cultures with M-F10 medium. Moreover, in Fig. 7, those cases with a higher proportion of $\mathrm{M} 3+$ cells tended to express the fragile site earlier than those with lower one, suggesting a possible effect of the cell proliferation on the expressivity. Thus, we consider that the non-correlation observed in Fig. 6 is only a reflection of other unknown factors which are also involved in the inter-individual variation of the expressivity.

By examining a large number of subjects, it was explored for the first time that there are some extents of correlation between the expression frequencies of a common fragile site by culture conditions of folate deprivation and APC treatment. It is generally considered that, at the fragile sites, the folate deficient condition induces a chromatid break through a thymidylate stress (Sutherland and Hecht, 1985), while the APC does through the inhibition of DNA polymerase- $\alpha$ (Glover et al., 1984). They are both involved in the DNA replication process, but with slightly different mechanisms. Thus the presently found positive correlation between the culture conditions led us to surmise that the inter-individual variation observed in the expressivity of a common fragile site may be ascribed at least partly to intrinsic variability in the DNA sequence of this specific site, that is probably caused by some physiological conditions of individuals.

Relevant to this point, the changing pattern of the expression frequency of fra(3)(p14) in successive days of M-F10 culture is very interesting (Fig. 7). Though in some cases the expressivity was elevated from 72 to $96 \mathrm{hr}$ of culture, in others it showed a peak in $72 \mathrm{hr}$ and decreased thereafter. It seems that a certain fraction of lymphocytes have a potency of expressing the fragile site. As mentioned above, the fraction must be circumscribed by some physiological conditions of host, while variable culture conditions as well as cell proliferation rate may influence it to emerge earlier or later in cultured cells. It is not sure now what kind of physiological trait affects the fragility of this specific site of chromosome. It is likely that the folate in the body plays a significant role in the fragility. Correlation of the expression of fra(3)(p14) to the plasma concentration of folate was very suggestive, but not entirely clear (Fig. 8). This might be due to the fact that the plasma concentration is a rather unstable variable, being altered with affection of certain life styles such as daily food consumption. Besides the folate, other unknown factors, such as steroid hormone, may also be involved in the mechanism of common fragile sites.

Acknowledgments This work was supported in part by a Grant-in-Aid for Cancer Research from the Ministry of Education, Science and Culture, by that from the Ministry of Health and Welfare, and by the Smoking Research Foundation in Japan.

Vol. 35, No. 4, 1990 
We are very grateful to Funabashi Blood Center for providing blood samples for this study.

\section{RFFFERENCES}

Craig-Holmes, A., Strong, L.C., Goodacre, A. and Pathak, S. 1987. Variation in the expression of aphidicolin-induced fragile sites in human lymphocyte cultures. Hum. Genet. 76: 134-137.

Dewdney, R.S., Lovell, D.P., Jenkinson, P.C. and Anderson, D. 1986. Variation in sister-chromatid exchange among 106 members of the general U.K. population. Mutation Res. 171: $43-51$.

D'Souza, D., Thomas, I.M. and Das, B.C. 1988. Variation in spontaneous chromosomal damage as a function of biologic rhythms in women. Hum. Genet. 79: 83-85.

Fuster, C., Miró, R., Templado, C., Barrios, L. and Egozcue, J. 1989. Expression of folate-sensitive fragile sites in lymphocyte chromosomes. Hum. Genet. 81: 243-246.

Glover, T.W., Berger, C., Coyle, J. and Echo, B. 1984. DNA polymerase inhibition by aphidicolin induces gaps and breaks at common fragile sites in human chromosomes. Hum. Genet. 67: $136-142$.

Hecht, F. 1988. Fragile site update. Cancer Genet. Cytogenet. 31: 125-128.

Kähkönen, M. 1988. Population cytogenetics of folate-sensitive fragile sites. I. Common fragile sites. Hum. Genet. 80: 344-348.

Morimoto, K., Sato, M. and Koizumi, A. 1983. Proliferative kinetics of human lymphocytes in culture measured by autoradiography and sister chromatid differential staining. Exp. Cell. Res. $145: 349-356$.

Murasko, D.M., Weiner, P. and Kaye, D. 1987. Decline in mitogen induced proliferation of lymphocytes with increasing age. Clin. Exp. Immunol. 70: 440-448.

Rao, P.N., Heerema, N.A. and Palmer, C.G. 1988a. Fragile sites induced by FUdR, caffeine, and aphidicolin. Hum. Genet. 78: 21-26.

Rao, P.N., Heerema, N.A. and Palmer, C.G. 1988b. Expression of fragile sites in childhood acute lymphoblastic leukemia patients and normal controls. Hum. Genet. 79: 329-334.

Smeets, D.F.C.M., Scheres, J.M.J.C. and Hustinx, T.W.J. 1986. The most common fragile site in man is 3p14. Hum. Genet. 72: 215-220.

Sugio, Y. and Kuroki, Y. 1989. Family study of common fragile sites. Hum. Genet. 82: 191193.

Sutherland, G.R. and Hecht, F. 1985. Fragile Sites on Human Chromosomes. Oxford University Press, New York, pp. 16-94.

Tahahashi, E., Hori, T. and Murata, M. 1988. Population cytogenetics of rare fragile sites in Japan. Hum. Genet. 78: 121-126.

Tice, R., Schneider, E.L., Kram, D. and Thorne, P. 1979. Cytokinetic analysis of the impaired proliferation response of peripheral lymphocytes from aged humans to phytohemagglutinin. J. Exp. Med. 149: 1029-1041. 